\title{
Combining TMS and EEG Offers New Prospects in Cognitive Neuroscience
}

\author{
Carlo Miniussi · Gregor Thut
}

Received: 25 November 2008/ Accepted: 27 January 2009/Published online: 25 February 2009

(C) Springer Science+Business Media, LLC 2009

\begin{abstract}
The combination of brain stimulation by transcranial magnetic stimulation (TMS) with simultaneous electroencephalographic (EEG) imaging has become feasible due to recent technical developments. The TMS-EEG integration provides real-time information on cortical reactivity and connectivity through the analysis of TMSevoked potentials (TEPs), and how functional activity links to behavior through the study of TMS-induced modulations thereof. It reveals how these effects vary as a function of neuronal state, differing between individuals and patient groups but also changing rapidly over time during task performance. This review discusses the wide range of possible TMS-EEG applications and what new information may be gained using this technique on the dynamics of brain functions, hierarchical organization, and cortical connectivity, as well as on TMS action per se. An advance in the understanding of these issues is timely and promises to have a substantial impact on many areas of clinical and basic neuroscience.
\end{abstract}

This is one of several papers published together in Brain Topography on the "Special Topic: TMS and EEG."

C. Miniussi $(\square)$

Department of Biomedical Sciences and Biotechnology,

National Institute of Neuroscience-Italy, University of Brescia,

Viale Europa 11, 25123 Brescia, Italy

e-mail: miniussi@med.unibs.it

C. Miniussi

Cognitive Neuroscience Section, IRCCS San Giovanni di Dio

Fatebenefratelli, Via Pilastroni 4, 25125 Brescia, Italy

G. Thut

Centre for Cognitive NeuroImaging, Department of Psychology, University of Glasgow, 58 Hillhead Street, Glasgow G12 8QB, UK
Keywords Transcranial magnetic stimulation (TMS) . Electroencephalography (EEG) $\cdot$ TMS-evoked potential (TEP) $\cdot$ Cognition · Functional imaging

\section{What Can the Combination of TMS and EEG Add to the Field of Cognitive Neuroscience?}

Recent years have seen the emergence of exciting new techniques for studying perceptual, motor and cognitive functions in the human brain. Transcranial Magnetic Stimulation (TMS) is an increasingly popular tool for mapping these processes. TMS uses a magnetic field for indirect electrical stimulation of the brain (Barker et al. 1985). The induced stimulation modifies activity in the neural tissue under the stimulating coil, leading to alterations in performance of a wide range of behavioral tasks (Pascual-Leone et al. 2000; Walsh and Cowey 2000; Walsh and Pascual-Leone 2003). In cognitive neuroscience, TMS is usually applied to one area at a time at a precise time point into a trial, in order to trace the time course of functionally relevant activity in the stimulated area. Thereby, TMS can be used as an interventional technique to investigate causality in the brain-behavior relationship. However, the effect of TMS on task performance does not necessarily reveal whether it is the stimulation of the target area that bears a direct causal relationship to execution of the task. The behavioral effects induced by TMS can be equivocal, in that performance in the same cognitive task can be shown to be either facilitated (Cappa et al. 2002) or inhibited (Shapiro et al. 2001) by TMS depending on the stimulated area, or in that the same stimulation parameters and site can have opposing effects on different tasks (Walsh et al. 1998; Rossi et al. 2001; Cappa et al. 2002). 
Such results not only highlight the complexity of TMS but also raise questions as to whether the observed effects result from stimulation of the target area or from some area "downstream" to the stimulated site. Another difficulty concerns the interpretation of a null result, where a task is unaffected by TMS of a specific area. A null result could mean that the stimulation truly had no effect or that the stimulation was insufficient to affect functioning in the target area. In addition, a null result may occur if two brain areas participate equally in the specified cognitive task, such that interfering with only one of them does not result in an overt behavioral change. Finally, a null result may arise from compensation during which another brain area takes over the respective function of the directly stimulated region. As demonstrated by (Sack et al. 2005), combining different TMS protocols at multiple sites allows for unmasking of the behavioral deficit by also blocking the compensation.

Whether TMS has a behavioral effect therefore depends on how it modifies the activity within the stimulated area and the network of cerebral areas that are functionally connected to the target region. In other words, since the function of a brain area is a property of the information processing circuits in which it participates (Passingham et al. 2002), TMSinduced changes in behavior may not only be ascribed to changes in excitability/activity of the area directly underneath the stimulating coil, but also to "secondary" effects on areas connected to the stimulated site (Sack and Linden 2003). Thus, behavioral effects induced by TMS can provide a proof of principle that the stimulated regions are critical to perform the task under investigation, but such observations do not clearly demonstrate how the stimulated area, or the involved circuit, has been modified.

A great advantage of functional neuroimaging is the ability to acquire measurements of activity in the entire brain, thus providing a broader picture of the cortical responses to TMS. Significant progress can be made to overcome the interpretational problems of TMS by combining it with EEG, as EEG captures the cortical activity corresponding to different stages of processing (i.e., from sensory coding over higher information processing to motor programming and output) with high temporal resolution.

As all neuroimaging techniques, EEG has its limitations. It only identifies correlational links between brain activity and behavior. Combining two different methods, such as TMS and EEG, has therefore also the advantage of overcoming the limitations of either technique alone, thereby supplementing the information provided by correlational analysis with a technique that can establish a causal link between brain function and behavior. Yet it should be recognized that the result of this integration is different from the sum of the two single techniques, and researchers should therefore take care in designing experiments, collecting, and interpreting data (see further aspects or problems to be considered).

In this work, we wish to provide an account of the advantages offered by combining these two ${ }^{1}$ methods, as well as to identify the potential problems in the interpretation of results so obtained.

\section{How TMS and EEG Can Be Integrated}

Recording EEG during TMS may be technically challenging, since TMS induces a very strong electrical field that can saturate recording amplifiers. However, advances in amplifier technology have led to the development of TMS-compatible EEG equipment that can work in very high, time-varying magnetic fields without saturation. Ilmoniemi et al. (1997; Virtanen et al. 1999) developed a TMS-compatible EEG system which includes gain-control and a sample-and-hold circuit that locks the EEG signal for several milliseconds immediately post-TMS. An alternative method has been implemented that uses slew-rate limited preamplifiers to prevent saturation during the TMS pulse, allowing continuous data recording and resulting in a short-lasting artifact (Thut et al. 2003, 2005; Ives et al. 2006). Finally, Bonato et al. (2006) have recently used TMS-compatible DC amplifiers with a wide dynamic range that do not require pinning of the preamplifiers, which allows continuous data recording without EEG signal saturation during TMS delivery.

\section{How TMS-EEG Integration Can be Used}

Event-related potentials obtained through EEG independently from TMS can be used to identify the time-window during which a given area is activated during performance of a cognitive task. This therefore provides information on when that area should be stimulated in order to investigate causal structure-function relationships, similar to the approach of using functional magnetic resonance imaging to define where exactly to stimulate (Sack et al. 2009). However, the combination of brain stimulation with simultaneous (on-line) electrophysiological imaging should go beyond this application and boost the amount of

\footnotetext{
${ }^{1}$ It is important to point out that these two techniques are far more complex than can be adequately discussed in this paper. TMS can be used in many different ways (e.g., as single or multiple pulses, at high or low frequency) and the outcome of TMS will depend on a number of technical variables, such as the frequency or intensity of stimulation and the timing of TMS application, i.e., before, in the initial phase, or in the final phase of the task. In the same way, EEG data can be analyzed with many different approaches, producing results that may reveal different sides of the same coin, although the relationship between them remains unclear.
} 
information that we can obtain by each method independently. TMS allows to present precisely timed stimulations to an anatomically or functionally localized region of the brain. It is thus possible to combine the precise timing of TMS with the high temporal resolution of EEG to disentangle the activation sequence of various cortical areas and their causal roles in the execution of cognitive tasks. Documenting changes across the brain by EEG in combination with TMS can therefore provide invaluable information about the dynamics of human brain function beyond what is possible by TMS or EEG alone.

In the following, we provide an overview on the wide range of possible TMS-EEG applications which we have grouped here in three approaches: (1) "inductive", in which the electrophysiological response generated by TMS is measured at the cortical level to assess cortical reactivity and connectivity across various conditions; (2) "interactive", which explores when, where, and how TMS affects a functional network during a cognitive task; and (3) "rhythmic", which uses TMS to interact with oscillatory brain activity in order to study causal relationships between cortical rhythms and perceptual, cognitive or motor processes. Further details on each of these three approaches has been provided previously [see reviews of (Komssi and Kahkonen 2006) taking into account inductive; of (Taylor et al. 2008) emphasizing interactive and of (Thut and Miniussi 2009) highlighting rhythmic approaches].

\section{Inductive: TMS-EEG to Explore the State} of Momentary Excitability of the Brain

TMS-evoked potentials (TEPs) recorded over the scalp are quantifiable markers of the cerebral neurophysiological state in behaviorally silent areas, in the same way as motor evoked potentials (MEP) recorded from muscles after TMS over motor cortex are markers of the state of the motor system. Several experiments on TEPs have demonstrated that it is possible to study the reactivity of the cortex in those areas that do not produce a peripheral marker of central excitability (Kahkonen et al. 2005), such as the motor cortex (MEP) or visual cortex (phosphenes), or for areas where a peripheral marker is present but the aim is to address the issue of cortical versus non cortical contributions to excitability changes (Nikulin et al. 2003). Therefore, evoked cortical reactivity can be used as a dependent variable in any experiment that plans to investigate the involvement of a cortical region in a given condition.

From a technical point of view, the inductive approach can be used to explore the most effective TMS parameters by systematically studying the consequences of modifying the characteristics of stimulation, e.g., the type of coil, the stimulation intensity, or the orientation of the coil (Komssi et al. 2004; Kahkonen et al. 2005; Bonato et al. 2006).
Typically, this can be done during what is termed "the resting state" (although a clear definition of "resting" has not been established). It has been shown that the reactivity of the motor cortex and that of the prefrontal cortex are related to TMS intensity, in that TEP amplitude increases with stimulus intensity (Komssi et al. 2004; Kahkonen et al. 2005). In addition to intensity, coil orientation has been shown to be an important factor in determining TEPs. TMS applied over the same target location with constant intensity but different degrees of coil orientation can modify TEP-amplitude (Bonato et al. 2006) as well as behavioral effects (Hill et al. 2000). This line of TMS-EEG research can thus provide new insights into technical aspects that are of importance to understand and fine tune TMS actions.

Another important area of investigation concerns the electrical response evoked by TMS in the stimulated area (reactivity) and connected sites (connectivity) (Ilmoniemi et al. 1997; Komssi et al. 2002, 2004; Kahkonen et al. 2005). In these experiments, no hypotheses are made about behavioral outcomes, and therefore the subjects' behavior is not evaluated. Rather, analysis is focused on the waveform, latency and cortical distribution of TEPs. These measures of cortical reactivity and connectivity depend on the physiological state of the neurons in the stimulated cortex (statedependency), as well as on the type of cortex stimulated (morphology). In a study on connectivity, Massimini and colleagues (Massimini et al. 2005) stimulated the premotor cortex of subjects while they were awake or sleeping. In both conditions, the local TEPs were similar, whereas remote responses differed dramatically. During wakefulness, TMSinduced activity spread within and between hemispheres, whereas during sleep, activity remained confined to regions surrounding the stimulated area. This illustrates that effective connectivity (i.e., interactions between distinct units within a nervous system) changes depending on the state of the brain (Massimini et al. 2005).

In short, the recording of TEPs provides valuable information on cortical reactivity and connectivity when compared across distinct conditions, such as before, during, and after a pharmacological or behavioral (rehabilitative) treatment in patients, or across different physiological or pathological states (Kahkonen and Ilmoniemi 2004; Komssi and Kahkonen 2006). Recording TEPs in the normal brain is therefore comparable to previous studies on sensory evoked potentials, providing normative data in controls that can be used for further research on pathophysiological conditions.

"Interactive": Using TMS-EEG to Explore the Functional and Hierarchical Dynamics of the Brain

TMS can be regarded to produce temporary functional modulations within neuronal networks involved in task 
execution. Therefore, it has been widely used to map the flow of information across different brain regions, allowing the identification of the cortical areas responsible for a given task. However, the mechanism of TMS modulations remains unclear and results should be interpreted with care. One has to distinguish between inhibitory/excitatory effects of TMS on neurons and negative/positive outcome on behavior, which can occur in any combination. In fact either type of neuronal change (excitatory or inhibitory) can induce either behavioral effect (negative or positive outcome). For instance, an excitatory effect of TMS on one area may (through inhibitory interconnections) induce inhibition of a different area that controls the cognitive task, resulting in a negative behavioral outcome. Alternatively, a local inhibitory effect may reduce the inhibition of the stimulated area on another, leading to facilitation of task performance (Sack and Linden 2003; Taylor et al. 2007a, b). Adding to this complexity is that the neural structures participating in these networks are flexibly linked, depending on the particular cognitive demands. It is also possible that the brain compensates for interference either within an area or across a circuit, since it does not react passively to cortical stimulation and because the state of activation influences the response (Silvanto et al. 2007; Romei et al. 2008a).

With these considerations in mind, TMS-EEG studies have set out to investigate where, when and how TMS interacts with task performance. In other words, EEG has been used to provide evidence about which area is affected by TMS (i.e., the stimulated area or a connected one), when in time the stimulation takes effect (i.e., at the moment of stimulation or later), and how the effects of TMS correlate with behavior (i.e., a cortical response congruent or incongruent with modified performance). Using the interactive application, we can therefore gain information not only on the causal role of a given area but also on its functional role with respect to other areas, making it possible to investigate its functionality from a network perspective.

Taylor and et al. (2007a) have shown that stimulation of the frontal eye field prior to the onset of a visual stimulus modulates the event-related cortical response recorded over posterior visual areas, demonstrating that TMS of one area affects functionally connected areas. The same group (Taylor et al. 2007b) also found that dorsal medial frontal cortex stimulation affected the lateralized readiness potentials recorded over hand motor areas during an action selection task (but see also Rossi et al. 2000). These effects were absent after stimulation of regions that have no connections with visual or motor cortices, and are thus likely to be mediated by interference with top-down control at higher levels of the functional hierarchy. These experiments are illustrative of manipulation of the activity of one area affecting another interconnected site.
The influence of TMS on the temporal sequence of visual responses has also been evaluated. While Taylor et al. (2007a) observed the effects on visual evoked potentials with FEF stimulation prior to the onset of the visual stimulus, Thut et al. (2003) showed changes of visual response at later time points post-stimulus onset with occipital stimulation. Single-pulse TMS was applied to the occipital cortex of several subjects viewing visual stimuli. The TMS pulse was delivered at the onset of the visual stimulus or soon after, when the visual cortex is supposed to reach peak activity (about $100 \mathrm{msec}$ after visual stimulus onset). Changes in the topography of the visual evoked potentials were observed only when TMS was applied during the build-up of the positive component known as P1 (about $100 \mathrm{msec}$ ). This provides direct clues to the temporal dynamics of how visual processing is altered by TMS.

In conclusion, TMS-EEG provides a unique opportunity to better understand the functional dynamics between different cortical areas of a network during task execution through interacting directly with the brain and recording the associated consequences on neural activity.

\section{"Rhythmic": Using TMS-EEG to Explore the Generation and Functional Role of Brain Rhythms}

The main advantage of this approach is the use of TMS within the broader perspective on brain rhythms that EEG provides. A consistent interest in the study of oscillatory brain activity in humans has emerged within the last decade (Singer 1999; Fries 2005; Schoffelen et al. 2005; Womelsdorf et al. 2006, 2007), with many studies having provided correlational evidence for activity in specific frequency bands to be linked to specific functions. Rhythmic TMS provides the opportunity to entrain brain oscillations, and, if behavioral modulation is concurrently observed, the opportunity to draw causal links between synchronization in functional networks and specific aspects of a task. Several studies have explored the possibility of inducing frequency specific effects by rhythmic TMS to modify cognition (Klimesch et al. 2003) and motor output measures (Brignani et al. 2008), showing that brain rhythms are causally implicated in shaping behavior. Others have used single-pulse TMS to define the relationship between the oscillatory state of the cortex and the response to a subsequent TMS pulse (Thut et al. 2006; Lepage et al. 2008; Romei et al. 2008a, b; Sauseng et al. 2008). Thus, EEG can be used to study how TMS interacts with rhythmic brain activity, and vice versa, as well as how rhythmic brain stimulation can be used to modify brain functions.

Given the recent advances in EEG research, the ability to modulate brain activity in the frequency domain is 
particularly relevant and promises to have a significant impact on many areas of clinical and basic neuroscience. The possibility of inducing neuromodulatory effects by rhythmic brain stimulation holds promise not only for advancing our understanding of brain rhythms but also in designing new neurorehabilitation strategies (Miniussi et al. 2008). Entraining specific oscillations in specific regions could develop into an effective application (see e.g., Dohrmann et al. 2007 for a promising neurofeedback application). A better understanding of the relationship between ongoing brain oscillations and rhythmic TMS thus represents an important step in the development of more adaptive neurorehabilitative strategies for directing the network toward a new pattern of activation via stimulation to improve cognitive dysfunction.

\section{Further Aspects or Problems to be Considered}

It is important to consider that TMS is inducing non specific or indirect responses in the brain, which may influence the EEG recording, besides modulating the electrical activity of the cortical areas involved in the task. These non specific, task unrelated contaminations consist of auditory responses (due to the coil click occurring concurrently with discharge of the magnetic stimulator) (Nikouline et al. 1999); of somatosensory responses (mostly due to trigeminal afferents or afferent responses after motor cortex stimulation) (Nikulin et al. 2003); of muscular responses (because of eye blink startle reflexes, eye movements induced by the coil click, or peripheral muscular contractions due to peripheral stimulation), or eventually movement of the electrodes due to coil vibration (Virtanen et al. 1999; Kahkonen et al. 2001, 2003). Also, general arousal due to TMS or auditory inter-sensory facilitation by the coil click (Marzi et al. 1998) might be present. All these effects should be eliminated or masked whenever possible. In instances where this is not possible, these artifacts should, as part of the experimental design, be reproduced in separate conditions (i.e., via control stimulation at appropriate sites), and their effects should be taken into account/parceled out during data analysis (Bender et al. 2005).

One data analysis strategy was proposed by Thut and colleagues for use with the interactive approach (Thut et al. 2003). To study TMS-induced changes on task related brain activity (visual evoked potentials in their case), the authors subtracted the non specific responses obtained through a condition of TMS without concurrent task from the main condition including TMS and task. In other words, to control for non specific TMS induced contaminations and artifacts, a control condition consisting of TMS only was used to build a template that was subtracted from the condition of TMS with the task (Thut et al. 2003, 2005). As was pointed out by the authors (Thut et al. 2005), such subtraction not only eliminates activity induced by TMS alone (the non specific contaminations and artifact) but also the (in this case) unwanted TMS induced cortical response (namely the TEP) which is expected to be present in both conditions (TMS alone and TMS and task). However, because the latter response is dependent on the physiological state of the neurons in the stimulated cortex, i.e., arises in interaction with ongoing activity at baseline or induced by the task, the result of the subtraction may not necessarily be directly comparable with the TMS-modified task-related activity; that is, the cortical response presumably correlating with the behavioral modification. Combining EEG and TMS does not simply correspond to the sum of the two techniques. Therefore, the cortical response elicited during TMS-EEG may not be the simple modification of a well known component, but may be a distinct electrophysiological response. Taylor et al. (2007a) noted that TMS to the frontal eye field induced a modification of behavioral responses that is similar to that observed when the subject is not paying attention to the stimulus. However, TMS did not lead to a cortical response similar to the one elicited by the task when the subject was not paying attention; rather, it resulted in a waveform with a different shape. The authors concluded that in these experiments, TMS did not reproduce, at the cortical level, the effect of behavioral inattention (Taylor et al. 2007a) but probably acted through a different mechanism. These considerations may also lead to the conclusion that direct comparison between experimental conditions, as opposed to subtraction of a baseline response, may be a better solution for identifying relative changes in the observed neural activity. Yet, even when comparing different experimental conditions, the alternative explanation, that differences are confounded by different TEPs, should be kept in mind when data are interpreted.

Because adequate modeling/reproduction of the nonspecific effects is of importance for the interpretation of TMS-EEG data, a significant impact on TMS-EEG research may come from the use of neuronavigation systems for precisely targeting a cortical region, thus allowing the researcher to exclude from the final analysis trials where TMS was not delivered within the target area or with a deviant coil orientation. Small variations in coil orientation and location can induce significant changes in behavior as well as cortical induced response (Lioumis et al. 2009). By this means, data can be filtered in a manner similar to the way many experiments on visual spatial attention reject trials in which the subject was not fixating on a given point.

Another promising TMS-EEG application is the modeling of the distribution of the induced current density in 
the brain to better understand and guide TMS administration (Ruohonen and Ilmoniemi 1999; Wagner et al. 2004). These applications can provide useful information about the stimulated structures in the brain, as well as allowing the calculation of the exact stimulation "dose" (i.e., strength of the electric field induced in the different areas of the cortex). This would permit the fine-tuning of stimulation based on anatomical characteristics of the subject rather than percentage of baseline reactivity/excitability (Ruohonen and Ilmoniemi 1999). In a spherical head model, the depth of TMS is limited since the magnetic field levels decrease rapidly with distance from the appliances. However, the capacity to stimulate deep structures depends on many factors including coil geometry, stimulus intensity and connectivity of the cortical area being stimulated. Computing the current density distribution induced in the cortex in a more realistic model can give important information on penetration and focus of stimulation, e.g., on how TMS takes effect in an atrophic brain as compared to a healthy brain (Wagner et al. 2006). In addition, through the comparison of maps representing the estimated TMSinduced electrical field and maps representing task-related activity, it becomes possible to gain a clearer picture of the levels of possible interactions between the two processes (i.e., overlap between cortical activity induced by the task and areas that are likely to be stimulated by TMS). Such a methodology can also be useful for learning more about how variations of stimulation characteristics can affect the system in different ways (pulse form, directing of induced current, intensity, site of stimulation to name a few). It is conceivable that TMS at low intensity stimulates the target area and functionally connected sites through cortico-cortical connections, while TMS at higher intensities may stimulate cortico-thalamic connections influencing functionally connected areas in a different way. We should also bear in mind that, during stimulation, we activate excitatory neurons/fibers as well as inhibitory neurons/fibers. The activation-to-inhibition ratio may depend on the depth of stimulation (affecting superficial vs. deep cortical layers) as well as the direction of the induced current. Therefore, combining modeling with actual measurements may prove of fundamental importance for the interpretation and understanding of TMS-actions.

\section{Concluding Remarks}

Neuroimaging studies have shown that TMS induces efficient and sometimes long-lasting modifications of cortical activity both locally (Paus et al. 2001; Lee et al. 2003; Pleger et al. 2006; Sack et al. 2007) and at distant sites (Pleger et al. 2006; Ruff et al. 2006; Sack et al. 2007; Ruff et al. 2008). Using the TMS-EEG integration, we can acquire information on the causal link between brain activity and function as well as on cortical reactivity and connectivity with other areas (via the interactive and inductive approaches respectively). The use of EEG to evaluate the effects induced by TMS may also assist in understanding the impact of temporal and spatial summation of pulses, in particular for a better understanding of oscillatory brain activity and its implication in functions (rhythmic approach). Finally, it is important to note that these studies can also help to identify the mechanisms of TMS action (Silvanto et al. 2007; Harris et al. 2008). This approach offers the potential to identify responses to TMS within an area or across circuits, thus helping to determine in vivo the neuronal processes affected, directly or indirectly, by magnetic stimulation.

Acknowledgements We thank Debora Brignani and Domenica Veniero for useful discussions.

\section{References}

Barker AT, Jalinous R, Freeston IL (1985) Non-invasive magnetic stimulation of human motor cortex. Lancet 1:1106-1107

Bender S, Basseler K, Sebastian I, Resch F, Kammer T, Oelkers-Ax R, Weisbrod M (2005) Electroencephalographic response to transcranial magnetic stimulation in children: evidence for giant inhibitory potentials. Ann Neurol 58:58-67

Bonato C, Miniussi C, Rossini PM (2006) Transcranial magnetic stimulation and cortical evoked potentials: a TMS/EEG coregistration study. Clin Neurophysiol 117:1699-1707

Brignani D, Manganotti P, Rossini PM, Miniussi C (2008) Modulation of cortical oscillatory activity during transcranial magnetic stimulation. Hum Brain Mapp 29:603-612

Cappa SF, Sandrini M, Rossini PM, Sosta K, Miniussi C (2002) The role of the left frontal lobe in action naming: rTMS evidence. Neurology 59:720-723

Dohrmann K, Weisz N, Schlee W, Hartmann T, Elbert T (2007) Neurofeedback for treating tinnitus. Prog Brain Res 1660:473-485

Fries P (2005) A mechanism for cognitive dynamics: neuronal communication through neuronal coherence. Trends Cogn Sci 9:474-480

Harris JA, Clifford CW, Miniussi C (2008) The functional effect of transcranial magnetic stimulation: signal suppression or neural noise generation? J Cogn Neurosci 20:734-740

Hill AC, Davey NJ, Kennard C (2000) Current orientation induced by magnetic stimulation influences a cognitive task. NeuroReport 11:3257-3259

Ilmoniemi RJ, Virtanen J, Ruohonen J, Karhu J, Aronen HJ, Naatanen R, Katila T (1997) Neuronal responses to magnetic stimulation reveal cortical reactivity and connectivity. NeuroReport 8:35373540

Ives JR, Rotenberg A, Poma R, Thut G, Pascual-Leone A (2006) Electroencephalographic recording during transcranial magnetic stimulation in humans and animals. Clin Neurophysiol 117:1870-1875

Kahkonen S, Ilmoniemi RJ (2004) Transcranial magnetic stimulation: applications for neuropsychopharmacology. J Psychopharmacol $18: 257-261$

Kahkonen S, Kesaniemi M, Nikouline VV, Karhu J, Ollikainen M, Holi M, Ilmoniemi RJ (2001) Ethanol modulates cortical 
activity: direct evidence with combined TMS and EEG. Neuroimage 14:322-328

Kahkonen S, Wilenius J, Nikulin VV, Ollikainen M, Ilmoniemi RJ (2003) Alcohol reduces prefrontal cortical excitability in humans: a combined TMS and EEG study. Neuropsychopharmacology 28:747-754

Kahkonen S, Komssi S, Wilenius J, Ilmoniemi RJ (2005) Prefrontal transcranial magnetic stimulation produces intensity-dependent EEG responses in humans. Neuroimage 24:955-960

Klimesch W, Sauseng P, Gerloff C (2003) Enhancing cognitive performance with repetitive transcranial magnetic stimulation at human individual alpha frequency. Eur J NeuroSci 17:1129-1133

Komssi S, Kahkonen S (2006) The novelty value of the combined use of electroencephalography and transcranial magnetic stimulation for neuroscience research. Brain Res Rev 52:183-192

Komssi S, Aronen HJ, Huttunen J, Kesaniemi M, Soinne L, Nikouline VV, Ollikainen M, Roine RO, Karhu J, Savolainen S, Ilmoniemi RJ (2002) Ipsi- and contralateral EEG reactions to transcranial magnetic stimulation. Clin Neurophysiol 113:175-184

Komssi S, Kahkonen S, Ilmoniemi RJ (2004) The effect of stimulus intensity on brain responses evoked by transcranial magnetic stimulation. Hum Brain Mapp 21:154-164

Lee L, Siebner HR, Rowe JB, Rizzo V, Rothwell JC, Frackowiak RS, Friston KJ (2003) Acute remapping within the motor system induced by low-frequency repetitive transcranial magnetic stimulation. J Neurosci 23:5308-5318

Lepage JF, Saint-Amour D, Théoret H (2008) EEG and neuronavigated single-pulse TMS in the study of the observation/ execution matching system: are both techniques measuring the same process? J Neurosci Methods 175:17-24

Lioumis P, Kicic D, Savolainen P, Makela JP, Kahkonen S (2009) Reproducibility of TMS-Evoked EEG responses. Hum Brain Mapp (in press)

Marzi CA, Miniussi C, Maravita A, Bertolasi L, Zanette G, Rothwell JC, Sanes JN (1998) Transcranial magnetic stimulation selectively impairs interhemispheric transfer of visuo-motor information in humans. Exp Brain Res 118:435-438

Massimini M, Ferrarelli F, Huber R, Esser SK, Singh H, Tononi G (2005) Breakdown of cortical effective connectivity during sleep. Science 309:2228-2232

Miniussi C, Cappa SF, Cohen LG, Floel A, Fregni F, Nitsche MA, Olivieri M, Pascual-Leone A, Paulus W, Priori A, Walsh V (2008) Efficacy of repetitive transcranial magnetic stimulation/ transcranial direct current stimulation in cognitive neurorehabilitation. Brain Stimulation 1:326-336

Nikouline V, Ruohonen J, Ilmoniemi RJ (1999) The role of the coil click in TMS assessed with simultaneous EEG. Clin Neurophysiol 110:1325-1328

Nikulin VV, Kicic D, Kahkonen S, Ilmoniemi RJ (2003) Modulation of electroencephalographic responses to transcranial magnetic stimulation: evidence for changes in cortical excitability related to movement. Eur J NeuroSci 18:1206-1212

Pascual-Leone A, Walsh V, Rothwell J (2000) Transcranial magnetic stimulation in cognitive neuroscience-virtual lesion, chronometry, and functional connectivity. Curr Opin Neurobiol 10:232-237

Passingham RE, Stephan KE, Kotter R (2002) The anatomical basis of functional localization in the cortex. Nat Rev Neurosci 3:606-616

Paus T, Sipila PK, Strafella AP (2001) Synchronization of neuronal activity in the human primary motor cortex by transcranial magnetic stimulation: an EEG study. J Neurophysiol 86:19831990

Pleger B, Blankenburg F, Bestmann S, Ruff CC, Wiech K, Stephan KE, Friston KJ, Dolan RJ (2006) Repetitive transcranial magnetic stimulation-induced changes in sensorimotor coupling parallel improvements of somatosensation in humans. J Neurosci 26:1945-1952
Romei V, Brodbeck V, Michel C, Amedi A, Pascual-Leone A, Thut G (2008a) Spontaneous fluctuations in posterior alpha-band EEG activity reflect variability in excitability of human visual areas. Cereb Cortex 18:2010-2018

Romei V, Rihs T, Brodbeck V, Thut G (2008b) Resting electroencephalogram alpha-power over posterior sites indexes baseline visual cortex excitability. NeuroReport 19:203-208

Rossi S, Pasqualetti P, Rossini PM, Feige B, Ulivelli M, Glocker FX, Battistini N, Lucking CH, Kristeva-Feige R (2000) Effects of repetitive transcranial magnetic stimulation on movement-relatedcortical activity in humans. Cereb Cortex 10:802-808

Rossi S, Cappa SF, Babiloni C, Pasqualetti P, Miniussi C, Carducci F, Babiloni F, Rossini PM (2001) Prefrontal cortex in long-term memory: an "interference" approach using magnetic stimulation. Nat Neurosci 4:948-952

Ruff CC, Blankenburg F, Bjoertomt O, Bestmann S, Freeman E, Haynes JD, Rees G, Josephs O, Deichmann R, Driver J (2006) Concurrent TMS-fMRI and psychophysics reveal frontal influences on human retinotopic visual cortex. Curr Biol 16:14791488

Ruff CC, Bestmann S, Blankenburg F, Bjoertomt O, Josephs O, Weiskopf N, Deichmann R, Driver J (2008) Distinct causal influences of parietal versus frontal areas on human visual cortex: evidence from concurrent TMS-fMRI. Cereb Cortex $18: 817-827$

Ruohonen J, Ilmoniemi RJ (1999) Modeling of the stimulating field generation in TMS. Electroencephalogr Clin Neurophysiol Suppl 51:30-40

Sack AT, Linden DE (2003) Combining transcranial magnetic stimulation and functional imaging in cognitive brain research: possibilities and limitations. Brain Res Brain Res Rev 43:41-56

Sack AT, Camprodon JA, Pascual-Leone A, Goebel R (2005) The dynamics of interhemispheric compensatory processes in mental imagery. Science 308:702-704

Sack AT, Kohler A, Bestmann S, Linden DE, Dechent P, Goebel R, Baudewig J (2007) Imaging the brain activity changes underlying impaired visuospatial judgments: simultaneous FMRI, TMS, and behavioral studies. Cereb Cortex 17:2841-2852

Sack AT, Kadosh RC, Schuhmann T, Moerel M, Walsh V, Goebel R (2009) Optimizing functional accuracy of tms in cognitive studies: a comparison of methods. J Cogn Neurosci (in press)

Sauseng P, Klimesch W, Gerloff C, Hummel FC (2008) Spontaneous locally restricted EEG alpha activity determines cortical excitability in the motor cortex. Neuropsychologia 47:31-44

Schoffelen JM, Oostenveld R, Fries P (2005) Neuronal coherence as a mechanism of effective corticospinal interaction. Science 308:111-113

Shapiro KA, Pascual-Leone A, Mottaghy FM, Gangitano M, Caramazza A (2001) Grammatical distinctions in the left frontal cortex. J Cogn Neurosci 13:713-720

Silvanto J, Muggleton NG, Cowey A, Walsh V (2007) Neural adaptation reveals state-dependent effects of transcranial magnetic stimulation. Eur J NeuroSci 25:1874-1881

Singer W (1999) Neuronal synchrony: a versatile code for the definition of relations? Neuron 24:49-65; 111-125

Taylor PC, Nobre AC, Rushworth MF (2007a) FEF TMS affects visual cortical activity. Cereb Cortex 17:391-399

Taylor PC, Nobre AC, Rushworth MF (2007b) Subsecond changes in top down control exerted by human medial frontal cortex during conflict and action selection: a combined transcranial magnetic stimulation electroencephalography study. J Neurosci 27:11343-11353

Taylor PC, Walsh V, Eimer M (2008) Combining TMS and EEG to study cognitive function and cortico-cortico interactions. Behav Brain Res 191:141-147

Thut G, Miniussi C (2009) New insights into rhythmic brain activity from TMS-EEG studies. Trends Cogn Sci (in press) 
Thut G, Northoff G, Ives JR, Kamitani Y, Pfennig A, Kampmann F, Schomer DL, Pascual-Leone A (2003) Effects of single-pulse transcranial magnetic stimulation (TMS) on functional brain activity: a combined event-related TMS and evoked potential study. Clin Neurophysiol 114:2071-2080

Thut G, Ives JR, Kampmann F, Pastor MA, Pascual-Leone A (2005) A new device and protocol for combining TMS and online recordings of EEG and evoked potentials. J Neurosci Methods 141:207-217

Thut G, Nietzel A, Brandt SA, Pascual-Leone A (2006) Alpha-band electroencephalographic activity over occipital cortex indexes visuospatial attention bias and predicts visual target detection. J Neurosci 26:9494-9502

Virtanen J, Ruohonen J, Naatanen R, Ilmoniemi RJ (1999) Instrumentation for the measurement of electric brain responses to transcranial magnetic stimulation. Med Biol Eng Comput 37:322-326

Wagner T, Gangitano M, Romero R, Theoret H, Kobayashi M, Anschel D, Ives J, Cuffin N, Schomer D, Pascual-Leone A (2004) Intracranial measurement of current densities induced by transcranial magnetic stimulation in the human brain. Neurosci Lett 354:91-94

Wagner T, Fregni F, Eden U, Ramos-Estebanez C, Grodzinsky A, Zahn M, Pascual-Leone A (2006) Transcranial magnetic stimulation and stroke: a computer-based human model study. Neuroimage 30:857-870

Walsh V, Cowey A (2000) Transcranial magnetic stimulation and cognitive neuroscience. Nat Rev Neurosci 1:73-79

Walsh V, Pascual-Leone A (2003) Transcranial magnetic stimulation: a neurochronometrics of mind. Mit Press, Cambridge, Massachusetts

Walsh V, Ellison A, Battelli L, Cowey A (1998) Task-specific impairments and enhancements induced by magnetic stimulation of human visual area V5. Proc Biol Sci 265:537-543

Womelsdorf T, Fries P, Mitra PP, Desimone R (2006) Gamma-band synchronization in visual cortex predicts speed of change detection. Nature 439:733-736

Womelsdorf T, Schoffelen JM, Oostenveld R, Singer W, Desimone R, Engel AK, Fries P (2007) Modulation of neuronal interactions through neuronal synchronization. Science 316:1609-1612 